\title{
A Single DBS-Lead to Stimulate the Thalamus and Subthalamus: Two-Story Targets for Tremor Disorders
}

\author{
Jumpei Sugiyama and Hiroki Toda* \\ Department of Neurosurgery, Tazuke Kofukai Medical Research Institute and Kitano Hospital, Osaka, Japan
}

Keywords: tremor, deep brain stimulation, ventral intermediate nucleus, ventral posterior oralis nucleus, thalamus, subthalamus

\section{OPEN ACCESS}

Edited by:

Takashi Morishita,

Fukuoka University, Japan

Reviewed by:

Arun Singh,

University of South Dakota,

United States

Konstantin Slavin,

University of Illinois at Chicago,

United States

*Correspondence:

Hiroki Toda

htoda-nsu@umin.ac.jp

Specialty section:

This article was submitted to

Motor Neuroscience,

a section of the journal

Frontiers in Human Neuroscience

Received: 07 October 2021 Accepted: 05 January 2022

Published: 24 January 2022

Citation:

Sugiyama J and Toda H (2022) A Single DBS-Lead to Stimulate the

Thalamus and Subthalamus:

Two-Story Targets for Tremor

Disorders.

Front. Hum. Neurosci. 16:790942. doi: 10.3389/fnhum.2022.790942

\section{INTRODUCTION}

The recent development of magnetic resonance-guided focused ultrasound highlights the clinical significance of the stereotactic surgery of the thalamus including deep brain stimulation (DBS). Essential tremor and parkinsonian tremor respond well to DBS of the ventral intermediate nucleus (Vim) of the thalamus (Cury et al., 2017; Dallapiazza et al., 2019). Other tremor disorders, such as dystonic tremor, multiple sclerosis tremor, and Holmes' tremor, are treated well with stimulation of the ventral oralis anterior (Voa) and posterior (Vop) nuclei of the thalamus (Katayama et al., 2005; Foote et al., 2006; Cury et al., 2017; Mongardi et al., 2020). The Voa/Vop is also chosen as a DBS target when an intraoperative Vim stimulation trial is not satisfactory (Katayama et al., 2005; Molnar et al., 2005). In such cases, the DBS electrode is placed slightly anteriorly to stimulate the Voa/Vop. The Vim and Voa/Vop are adjacent primary targets to treat various tremor disorders.

The subthalamus, located beneath the ventral tier of the thalamic motor nuclei, also contains targets to control tremor disorders. The known surgical targets within the subthalamus are the caudal part of the zona incerta (Zi, cZi) (Fytagoridis et al., 2012; Eisinger et al., 2018), prelemniscal radiation (Raprl) (Kitagawa et al., 2000; Castro et al., 2015), and Fields H of Forel (Contreras Lopez et al., 2016; Fleury et al., 2016). The cZI and Raprl are often referred as the posterior subthalamic area (PSA) (Blomstedt et al., 2009).

Some refractory tremors may need two DBS-leads per hemisphere to stimulate dual- targets within the thalamus and subthalamus (Foote et al., 2006; Kobayashi et al., 2014). While two leads can generate a complex volume of tissue activated (VAT) and stimulate both pallido-thalamic and cerebello-thalamic projections, the multiple-lead insertion may increase the risk of surgical morbidity and dual internal pulse generators may be implanted per side.

The technological advances in the last 10 years include new DBS-lead designs as eight-contact directional or long span lead and programming technologies as interleaved or independent current control systems. With these advances, we can design complex volumes of tissue activated and apply these technologies for a single DBS-lead dual-target approach. This opinion article describes the benefit of a single DBS-lead trajectory going through two-story targets made up of the ventral motor nuclei of the thalamus (upstairs) and subthalamus (downstairs). 


\section{DBS TARGET AND TECHNOLOGY DBS Target Anatomy \\ Ventral Motor Nuclei of the Thalamus}

Above the plane containing the anterior commissure (AC) and posterior commissure (PC), the ventral motor nuclei of the thalamus are arrayed as Voa, Vop, and Vim from the rostral to caudal direction (Figure 1A). The ventral tier of these thalamic ventral nuclei is around the AC-PC plane. The Vim is about $5-8 \mathrm{~mm}$ anterior to the PC (Guiot et al., 1961; Ohye et al., 1982; Jankovic et al., 1995) and is located anterior to the ventral caudal nucleus $(\mathrm{Vc})$ which receives the sensory spinothalamic projection. The anteroposterior length of the Vim is $2-3 \mathrm{~mm}$ (Schaltenbrand et al., 1977). The Vim mainly receives the cerebellar projection via the fasciculus cerebello-thalamicus contained in the Raprl. A minor portion of the fasciculus thalamics from the pallidal component projects into the Vim (Gallay et al., 2008).

The Voa and Vop are located anterior to the Vim. The anteroposterior lengths of the Voa and Vop are both 1.5-2 mm (Schaltenbrand et al., 1977). The Voa/Vop receives most pallidothalamic projection via the fasciculus thalamicus ( $f t$, Figure 1B) (Gallay et al., 2008). The $f t$ are made up of the ansa lenticularis $(a l)$ and fasciculus lenticularis $(f l)$. The al originates from the ventral globus pallidus interna (GPi). The $a l$ is ventral and medial of the subthalamic nucleus (STN) and makes a turn (ansa) to go over the STN. The $f l$ originates from the dorsal GPi. The $f l$ goes between the dorsal STN and ventral $\mathrm{Zi}$, the field $\mathrm{H} 2$ of Forel (Forel H2). The $a l$ and $f l$ go through the prerubral field $\mathrm{H}$ of Forel (Forel $\mathrm{H}$ ) and merge to become the $f t$. The $f t$ goes through field $\mathrm{H} 1$ of Forel (Forel H1) and then enters the Voa/Vop (Gallay et al., 2008).

\section{Subthalamus}

The subthalamus is located underneath the AC-PC plane and beneath the ventral border of the thalamic motor nuclei (Parent and Carpenter, 1996). The subthalamus can be divided as the anterior and posterior subthalamic areas (Blomstedt et al., 2009). In the anterior subthalamic area, there are four layers from the dorsal to ventral direction: (i) Forel H1(including the $f t$ as described above), (ii) rostal $\mathrm{Zi}$, (iii) Forel H2 (including $f$ ), and (iv) STN. The posterior subthalamic area (PSA) consists of the cZi and Raprl (Figure 1A) (Blomstedt et al., 2009). The PSA is bounded by the STN (anterior), medial lemniscus (posterior), red nucleus (medial), and internal capsule and the $\mathrm{Vc}$ of the thalamus (lateral). The $\mathrm{Zi}$ has broad bidirectional projections to the cerebellum, brainstem reticular formation, pedunculopontine nucleus, substantia nigra pars reticulata, GPi, superior colliculus, cerebral cortex, and spinal cord. Most Zi neurons are inhibitory GABAergic neurons and include excitatory glutamatergic and dopaminergic neurons (Wang et al., 2020). The Raprl is constituted of cerebellothalamic tract and mesencephaloreticulo-thalamic tract (Gallay et al., 2008; Blomstedt et al., 2009, 2012; Ramirez-Zamora et al., 2016; Nowacki et al., 2018).

\section{DBS Trajectory}

A single DBS-lead trajectory can align the thalamic and subthalamic targets by adjusting the entry point and trajectory angle. The combinations of the thalamic and subthalamic targets include Voa/Vop-Vim, Vop-subthalamus, and Vimsubthalamus (Figure 1A). The posterior subthalamic area can be localized according to the red nucleus. Slight adjustment of the mediolateral angle may choose the $\mathrm{CZi}$ in the lateral PSA and the Raprl in the posteromedial PSA (inset of Figure 1A).

\section{DISCUSSION}

The dual-target approach can be a useful strategy to treat refractory tremors such as Holmes' tremor (Foote and Okun, 2005; Foote et al., 2006; Kobayashi et al., 2014; Artusi et al., 2018; Parker et al., 2020). Holmes' tremor manifests a mixed presentation of basal ganglia tremor via the pallido-thalamic projection and cerebellar tremor via the cerebello-thalamic projection (Deuschl et al., 2001). Therefore, Holmes' tremor may be treated with dual-target strategy stimulating the Voa/Vop and the Vim nuclei by using dual DBS-lead insertion (Foote and Okun, 2005; Foote et al., 2006; Kobayashi et al., 2014; Artusi et al., 2018; Parker et al., 2020).

A single DBS-lead dual-target approach is an alternative to the dual DBS-lead insertion. Several groups, including the authors, utilize the single DBS-lead approach to stimulate both striato-pallido-thalamic and cerebello-thalamic projections for patients with essential tremor (Barbe et al., 2018; Bot et al., 2018; Dos Santos Ghilardi et al., 2018), Holmes' tremor (Toda et al., 2017b), and atypical tremor (Toda et al., 2017a; Nakajima et al., 2021). Placing the single DBS-lead across the Vop/Vim border is the simplest option to stimulate both the striato-pallidal and cerebellar pathways (Nakajima et al., 2021). The Vim (Toda et al., 2017a; Barbe et al., 2018; Bot et al., 2018; Dos Santos Ghilardi et al., 2018) and the Voa/Vop nuclei (Toda et al., 2017b; Parker et al., 2020) are also aligned with the PSA on a single trajectory (Figure 1A). As traditional and empirical surgical nuances for Vim electrode placement, some surgeons insert the electrode slightly deeper in the ventral border of the thalamus to stimulate subthalamus as well as Vim. Nowadays, the long DBS octrode can traverse the ventral tier of the thalamus and subthalamus (Dos Santos Ghilardi et al., 2018). A recent trial by the University of Cologne (Barbe et al., 2018) compares stimulation of the Vim and PSA by inserting the single lead traversing both targets and concludes the equal and possibly more efficient effect of PSA-DBS than Vim-DBS. The best electrode to control tremors may vary among patients. Therefore, the clinician can compare the effects of the Vim stimulation and the PSA stimulation by selecting the electrode along the single DBS lead (Barbe et al., 2018; Dos Santos Ghilardi et al., 2018).

In addition, the interleaved (Toda et al., 2017a,b) or multiple independent current control system can expand the benefit of single lead stimulation by stimulating multiple electrodes along the lead. Therefore, even with the single lead insertion, patient and clinician can have options for stimulating multiple targets to control refractory tremors with no additional DBS lead. 
A

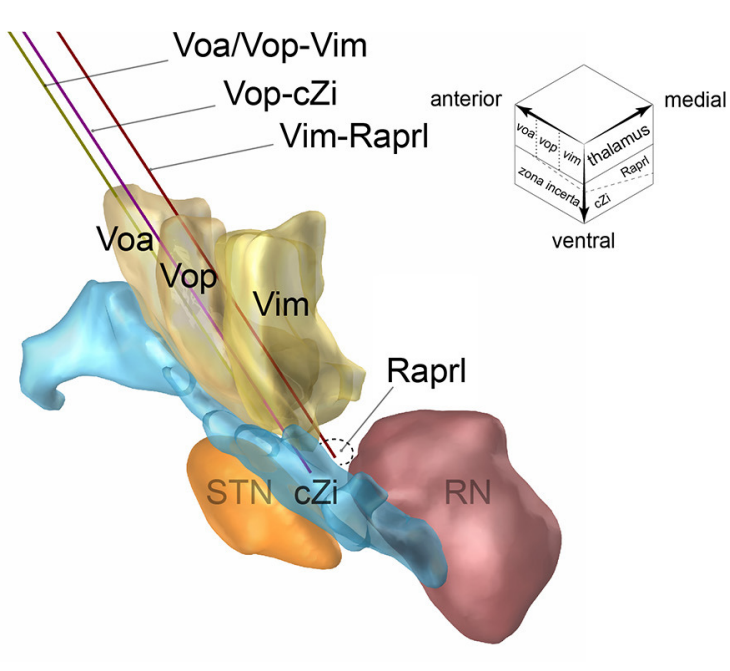

\section{B}

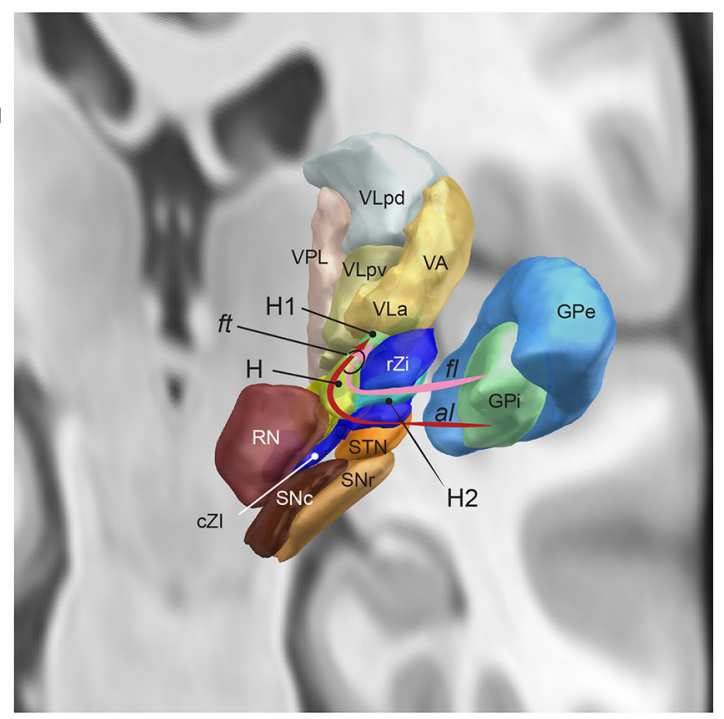

FIGURE 1 | (A) The single DBS-lead dual-target trajectories going through two-story targets made of the ventral thalamic nuclei (upstairs) and posterior subthalamic area (downstairs, inset): VoaNop-Vim (yellow), Vop-cZi (blue), and Vop-Raprl (brown). Voa, nucleus ventralis oralis anterior; Vop, nucleus ventralis oralis posterior; Vim, nucleus ventralis intermedius; cZi, caudal zona incerta; Raprl, prelemniscal radiation; RN, nucleus ruber; STN, nucleus subthalamicus. The upper right voxel scheme illustrates the two-story structure of ventral motor nuclei of the thalamus (upstairs) and subthalamus (downstairs). (B) Fasciculus lenticularis ( $f$ ) leaves from dorsal globus pallidus interna (GPi), enters the field $\mathrm{H} 2$ of Forel $(\mathrm{H} 2)$ between rostral zona incerta ( $r$ Zi) and the subthalamic nucleus (STN), joined with ansa lenticularis(al) from ventral GPi in the field $\mathrm{H}$ of Forel $(\mathrm{H})$, rostral to the red nucleus (RN), and form the fasciculus thalamicus ( $f t)$ in the field $\mathrm{H} 1$ of Forel $(\mathrm{H})$ then projects to the VA, and VLa nuclei of the thalamus. GPe, GP externa; SNc substantia nigra pars compacta; SNr, SN pars reticulata; VLpd and VLpv, ventrolateral posterior dorsal and ventral nuclei of the thalamus; VPL, ventroposterolateral nucleus of the thalamus [Reproduced from Toda et al. (2021) with permission from the Igaku-Shoin, Ltd., Japan]. The figure components are generated using Lead-DBS (https://www.lead-dbs.org/).

Furthermore, PSA components can be stimulated selectively when the lead is placed at the border of the Raprl and cZi. The directional lead can also stimulate the cZi, Raprl, or both by choosing directed electrodes. Further complex insertion is possible by careful preoperative imaging examination. The recent postoperative imaging technology as Lead-DBS [https://www. lead-dbs.org/, (Horn et al., 2019)] can be a useful investigational tool to estimate and predict the deep brain structure along the DBS lead.

The single DBS-lead dual-target approach can be applied bilaterally to control tremors on both limbs, especially for patients with essential tremor (Barbe et al., 2018; Bot et al., 2018; Dos Santos Ghilardi et al., 2018). In addition, the bilateral dualtarget strategy can be useful for midline tremor. The authors utilized bilateral Vim-PSA DBS leads to control tongue tremor and atypical parkinsonism which manifests as rigidity of the bilateral limbs (Toda et al., 2017a).

Application of the single-lead dual target approach is limited by the presence of cortical vein, lateral ventricle, and intraparenchymal vessels along the trajectory. Therefore, the detailed planning of the trajectory and target coordinates is necessary for single lead insertion for dual target.

In conclusion, the single DBS-lead dual-target stimulation within the ventral tier of the motor thalamic nuclei and the subthalamus can be a useful solution for refractory tremor.

\section{AUTHOR CONTRIBUTIONS}

All authors contributed to manuscript conception, draft, writing, revision, read, and approved the submitted version.

\section{FUNDING}

HT received Grant-in-Aid for Scientific Research (21K09116) for this research from Japan Society for the Promotion of Science.

\section{ACKNOWLEDGMENTS}

The authors acknowledge Dr. Ryosuke Okumura (Department of Radiology) for their generous support in radiological imaging.

\section{REFERENCES}

Artusi, C. A., Farooqi, A., Romagnolo, A., Marsili, L., Balestrino, R., Sokol, L. L., et al. (2018). Deep brain stimulation in uncommon tremor

disorders: indications, targets, and programming. J. Neurol. 265, 2473-2493. doi: 10.1007/s00415-018-8823-x

Barbe, M. T., Reker, P., Hamacher, S., Franklin, J., Kraus, D., Dembek, T. A., et al. (2018). DBS of the PSA and the VIM in essential tremor: 
a randomized, double-blind, crossover trial. Neurology 91, e543-e550. doi: 10.1212/WNL.0000000000005956

Blomstedt, P., Fytagoridis, A., Åström, M., Linder, J., Forsgren, L., and Hariz, M. I. (2012). Unilateral caudal zona incerta deep brain stimulation for Parkinsonian tremor. Parkinsonism and related disorders 18, 1062-1066. doi: 10.1016/j.parkreldis.2012.05.024

Blomstedt, P., Sandvik, U., Fytagoridis, A., and Tisch, S. (2009). The posterior subthalamic area in the treatment of movement disorders: past, present, and future. Neurosurgery 64, 1029-1038; discussion 1038-42. doi: 10.1227/01.NEU.0000345643.69486.BC

Bot, M., van Rootselaar, F., Contarino, M. F., Odekerken, V., Dijk, J., de Bie, R., et al. (2018). Deep brain stimulation for essential tremor: aligning thalamic and posterior subthalamic targets in 1 surgical trajectory. Oper Neurosurg. 15, 144-152. doi: 10.1093/ons/opx232

Castro, G., Carrillo-Ruiz, J. D., Salcido, V., Soto, J., García-Gomar, G., Velasco, A. L., et al. (2015). Optimizing prelemniscal radiations as a target for motor symptoms in Parkinson's disease treatment. Stereotact. Funct. Neurosurg. 93, 282-291. doi: 10.1159/000433446

Contreras Lopez, W. O., Azevedo, A. R., Cury, R. G., Alencar, F., Neville, I. S., Reis, P. R., et al. (2016). Incerta/VOP radiofrequency lesioning guided by combined stereotactic mri and microelectrode recording for posttraumatic midbrain resting-kinetic tremor. World Neurosurg. 86, 316-320. doi: 10.1016/j.wneu.2015.09.031

Cury, R. G., Fraix, V., Castrioto, A., Pérez Fernández, M. A., Krack, P., Chabardes, S., et al. (2017). Thalamic deep brain stimulation for tremor in Parkinson disease, essential tremor, and dystonia. Neurology 89, 1416-1423. doi: 10.1212/WNL.0000000000004295

Dallapiazza, R. F., Lee, D. J., De Vloo, P., Fomenko, A., Hamani, C., Hodaie, M., et al. (2019). Outcomes from stereotactic surgery for essential tremor. J. Neurol Neurosurg. Psychiatry 90, 474-482. doi: 10.1136/jnnp-2018-318240

Deuschl, G., Raethjen, J., Lindemann, M., and Krack, P. (2001). The pathophysiology of tremor. Muscle Nerve 24, 716-735. doi: 10.1002/mus. 1063

Dos Santos Ghilardi, M. G., Ibarra, M., Alho, E. J. L., Reis, P. R., Lopez Contreras, W. O., Hamani, C., et al. (2018). Double-target DBS for essential tremor: 8contact lead for cZI and Vim aligned in the same trajectory. Neurology 90, 476-478. doi: 10.1212/WNL.0000000000005076

Eisinger, R. S., Wong, J., Almeida, L., Ramirez-Zamora, A., Cagle, J. N., Giugni, J. C., et al. (2018). Ventral intermediate nucleus versus zona incerta region deep brain stimulation in essential tremor. Mov. Disord. Clin. Pract. 5, 75-82. doi: $10.1002 / \mathrm{mdc} 3.12565$

Fleury, V., Pollak, P., Gere, J., Tommasi, G., Romito, L., Combescure, C., et al. (2016). Subthalamic stimulation may inhibit the beneficial effects of levodopa on akinesia and gait. Mov. Disord. 31, 1389-1397. doi: 10.1002/mds.26545

Foote, K. D., and Okun, M. S. (2005). Ventralis intermedius plus ventralis oralis anterior and posterior deep brain stimulation for posttraumatic holmes tremor: two leads may be better than one: technical note. Neurosurgery 56:E445. doi: 10.1227/01.NEU.0000157104.87448.78

Foote, K. D., Seignourel, P., Fernandez, H. H., Romrell, J., Whidden, E., Jacobson, C., et al. (2006). Dual electrode thalamic deep brain stimulation for the treatment of posttraumatic and multiple sclerosis tremor. Neurosurgery 58:ONS-280-5. doi: 10.1227/01.NEU.0000192692.95455.FD

Fytagoridis, A., Sandvik, U., Aström, M., Bergenheim, T., and Blomstedt, P. (2012). Long term follow-up of deep brain stimulation of the caudal zona incerta for essential tremor. J. Neurol. Neurosurg. Psychiatry 83, 258-262. doi: 10.1136/jnnp-2011-300765

Gallay, M. N., Jeanmonod, D., Liu, J., and Morel, A. (2008). Human pallidothalamic and cerebellothalamic tracts: anatomical basis for functional stereotactic neurosurgery. Brain Struct. Funct. 212, 443-463. doi: 10.1007/s00429-007-0170-0

Guiot, G., Hertzog, E., Rondot, P., and Molina, P. (1961). Arrest or acceleration of speech evoked by thalamic stimulation in the course of stereotaxic procedures for Parkinsonism. Brain 84, 363-379. doi: 10.1093/brain/84.3.363

Horn, A., Li, N., Dembek, T. A., Kappel, A., Boulay, C., Ewert, S., et al. (2019). LeadDBS v2: towards a comprehensive pipeline for deep brain stimulation imaging. NeuroImage 184, 293-316. doi: 10.1016/j.neuroimage.2018.08.068

Jankovic, J., Cardoso, F., Grossman, R. G., and Hamilton, W. J. (1995). Outcome after stereotactic thalamotomy for parkinsonian, essential, and other types of tremor. Neurosurgery 37, 680-6; discussion 686-7. doi: 10.1097/00006123-199510000-00011

Katayama, Y., Kano, T., Kobayashi, K., Oshima, H., Fukaya, C., and Yamamoto, T. (2005). Difference in surgical strategies between thalamotomy and thalamic deep brain stimulation for tremor control. J. Neurol. 252 (Suppl. 4), Iv17-Iv22. doi: 10.1007/s00415-005-4005-8

Kitagawa, M., Murata, J., Kikuchi, S., Sawamura, Y., Saito, H., Sasaki, H., et al. (2000). Deep brain stimulation of subthalamic area for severe proximal tremor. Neurology 55, 114-116. doi: 10.1212/WNL.55.1.114

Kobayashi, K., Katayama, Y., Oshima, H., Watanabe, M., Sumi, K., Obuchi, T., et al. (2014). Multitarget, dual-electrode deep brain stimulation of the thalamus and subthalamic area for treatment of Holmes' tremor. J. Neurosurg. 120, 1025-1032. doi: 10.3171/2014.1.JNS12392

Molnar, G. F., Pilliar, A., Lozano, A. M., and Dostrovsky, J. O. (2005). Differences in neuronal firing rates in pallidal and cerebellar receiving areas of thalamus in patients with Parkinson's disease, essential tremor, and pain. J. Neurophysiol. 93, 3094-3101. doi: 10.1152/jn.00881.2004

Mongardi, L., Rispoli, V., Scerrati, A., Giordano, F., Capone, J. G., Vaudano, A. E., et al. (2020). brain stimulation of the ventralis oralis anterior thalamic nucleus is effective for dystonic tremor. Parkinsonism Relat. Disord. 81, 8-11. doi: 10.1016/j.parkreldis.2020.0 9.040

Nakajima, Y., Kambe, D., Toda, H., Nishida, N., Nagao, S., Sawamoto, N., et al. (2021). Thalamic deep brain stimulation for refractory atypical tremor after encephalitis of unknown etiology: a case report. NMC Case Rep. J. 8, 247-252. doi: 10.2176/nmccrj.cr.20200245

Nowacki, A., Debove, I., Rossi, F., Schlaeppi, J. A., Petermann, K., Wiest, R., et al. (2018). Targeting the posterior subthalamic area for essential tremor: proposal for MRI-based anatomical landmarks. J. Neurosurg. 131, 820-827. doi: 10.3171/2018.4.JNS1 8373

Ohye, C., Miyazaki, M., Hirai, T., Shibazaki, T., Nakajima, H., and Nagaseki, Y. (1982). Primary writing tremor treated by stereotactic selective thalamotomy. J. Neurol. Neurosurg. Psychiatry 45, 988-997. doi: 10.1136/jnnp.45.1 1.988

Parent, A., and Carpenter, M. B. (1996). Carpenter's Human Neuroanatomy. Media, PA: Williams and Wilkins.

Parker, T., and Raghu, A. L. B., FitzGerald, J. J., Green, A. L., Aziz, T. Z. (2020). Multitarget deep brain stimulation for clinically complex movement disorders. J. Neurosurg. 1-6. doi: 10.3171/2019.11.JNS19 2224

Ramirez-Zamora, A., Smith, H., Kumar, V., Prusik, J., Phookan, S., and Pilitsis, J. G. (2016). Evolving concepts in posterior subthalamic area deep brain stimulation for treatment of tremor: surgical neuroanatomy and practical considerations. Stereotact. Funct. Neurosurg. 94, 283-297. doi: 10.1159/00044 9007

Schaltenbrand, G., Wahren, W., and Hassler, R. (1977). Atlas for Stereotaxy of the Human Brain. Stuttgart: Thieme.

Toda, H., Asanuma, K., Kondo, T., Terada, Y., and Saiki, H. (2017a). Tongue tremor as a manifestation of atypical Parkinsonism treated with coaxial deep brain stimulation of thalamus and subthalamic area. Parkinsonism Relat. Disord. 44, 154-156. doi: 10.1016/j.parkreldis.2017.0 8.023

Toda, H., Kambe, D., Shima, A., Nishida, N., and Sawamoto, N. (2021). [Posterior subthalamic area, pallidothalamic tract, and pedunculopontine nucleus:deep brain stimulation targets for Parkinson's disease and essential tremor]. No Shinkei Geka 49, 820-828. doi: 10.11477/mf.143620 4460

Toda, H., Nishida, N., and Iwasaki, K. (2017b). Coaxial interleaved stimulation of the thalamus and subthalamus for treatment of Holmes tremor. Neurosurg. Focus 42:V1. doi: 10.3171/2017.4.FocusVid.1 6510

Wang, X., Chou, X. L., Zhang, L. I., and Tao, H. W. (2020). Zona incerta: an integrative node for global behavioral modulation. Trends Neurosci. 43, 82-87. doi: 10.1016/j.tins.2019.1 1.007 
Conflict of Interest: The authors declare that the research was conducted in the absence of any commercial or financial relationships that could be construed as a potential conflict of interest.

Publisher's Note: All claims expressed in this article are solely those of the authors and do not necessarily represent those of their affiliated organizations, or those of the publisher, the editors and the reviewers. Any product that may be evaluated in this article, or claim that may be made by its manufacturer, is not guaranteed or endorsed by the publisher.

Copyright (c) 2022 Sugiyama and Toda. This is an open-access article distributed under the terms of the Creative Commons Attribution License (CC BY). The use, distribution or reproduction in other forums is permitted, provided the original author(s) and the copyright owner(s) are credited and that the original publication in this journal is cited, in accordance with accepted academic practice. No use, distribution or reproduction is permitted which does not comply with these terms. 